\title{
Preclinical animal research on therapy dosimetry with dual isotopes
}

\author{
Mark W. Konijnenberg • Marion de Jong
}

Received: 28 January 2011 / Accepted: 22 February 2011 /Published online: 1 April 2011

(C) The Author(s) 2011. This article is published with open access at Springerlink.com

\begin{abstract}
Preclinical research into radionuclide therapies based on radiation dosimetry will enable the use of any LETequivalent radionuclide. Radiation dose and dose rate have significant influence on dose effects in the tumour depending on its radiation sensitivity, possibilities for repair of sublethal damage, and repopulation during or after the therapy. Models for radiation response of preclinical tumour models after peptide receptor radionuclide therapy based on the linear quadratic model are presented. The accuracy of the radiation dose is very important for observation of dose-effects. Uncertainties in the radiation dose estimation arise from incomplete assay of the kinetics, low accuracy in volume measurements and absorbed dose S-values for stylized models instead of the actual animal geometry. Normal dose uncertainties in the order of $20 \%$ might easily make the difference between seeing a dose-effect or missing it altogether. This is true for the theoretical case of a homogeneous tumour type behaving in vivo in the same way as its cells do in vitro. Heterogeneity of tumours induces variations in clonogenic cell density, radiation sensitivity, repopulation capacity and repair kinetics. The influence of these aspects are analysed within the linear quadratic model for tumour response to radionuclide therapy. Preclinical tumour models tend to be less heterogenic than the clinical conditions they should represent. The results of various preclinical radionuclide therapy experiments for peptide receptor radionuclide therapy are compared to the outcome of theoretical models and the influence of increased heterogeneity is analysed when the results of preclinical research is transferred to the clinic. When the radiation dose and radiobiology of the tumour response is known well enough it may be possible to leave the current phenomenological approach in preclinical radionuclide therapy
\end{abstract}

\footnotetext{
M. W. Konijnenberg $(\bowtie) \cdot$ M. de Jong

Nuclear Medicine Department, Erasmus MC,

Rotterdam, The Netherlands

e-mail: m.konijnenberg@erasmusmc.nl
}

and start basing these experiments on radiation dose. Then the use of a gamma ray-emitting radionuclides for a chemically comparable beta-particle-emitting paired isotope for therapy evaluation would be feasible.

Keywords Radiation dosimetry - Dose-effect models . Radionuclide therapy

\section{Introduction}

Preclinical research into radionuclide therapy has only recently shifted gear when specific animal dose models were introduced and evaluation of dosimetry and dose-effect models are increasingly being performed and consequently dose-effect relations are now being investigated [1-4]. The research into dose-effect models for radionuclide therapies is mainly aimed at reducing radiation toxicity in normal organs which show physiological uptake of the radiolabelled compound. The efficacy of radionuclide therapies is mainly studied at a phenomenological level: a practical amount of radioactivity is used and the reduction in tumour size observed, while keeping the radiation toxicity manageable.

The therapeutic effect of radionuclide therapy has been tested in mice and rats in various settings [5]. The response of tumours has scarcely been studied in relation to the radiation dose given, and is usually reported in relation to the activity administered. When radiation doses are reported at all it is usually for a single type of administration, making a dynamic interpretation of the dose-effect relationship by, for example, fractionation difficult to interpret. Tumour growth suppression of 4-5 weeks has been reported by Behr et al. after administration of $8-10$ Gy by ${ }^{90}$ Y-labelled CEA antibody fragments in xenografted mice [6]. Higher tumour doses shifted the suppression further, but renal and haemato- 
logical toxicity prevented further escalation of the dose. Different dosimetry models were tested ranging from spheres with just self-doses with activity inside the tumour or organ, to a cylindrical mouse model [4] with cross-organ dose contributions, to a realistic mouse model based on ultrasound imaging geometry. The choice of dose model influenced, for example, the kidney dose by more than $50 \%$ [6].

Peptide receptor radionuclide therapy is based on dose-driven preclinical experiments. Rats with subcutaneously implanted somatostatin receptor-positive CA20948 tumours were injected with ${ }^{90}$ Y-DOTA,Tyr ${ }^{3}$-octreotide [7] or with ${ }^{177}$ Lu-DOTA,Tyr ${ }^{3}$ octreotate [8]. A difference in tumour killing efficacy was observed between the responses of tumours of different size. An activity of $370 \mathrm{MBq}{ }^{90} \mathrm{Y}$-DOTA,Tyr ${ }^{3}$-octreotide led to complete cure only in tumours with a surface area of $3-9 \mathrm{~cm}^{2}$, whereas this activity led to only a partial response and prolongation of survival times in smaller tumours. With ${ }^{177}$ Lu-DOTA,Tyr ${ }^{3}$-octreotate, on the other hand, two doses of 277.5 MBq led to a complete response in small tumours with a surface area less than $1 \mathrm{~cm}^{2}$. Dosimetry was reported for both situations: $370 \mathrm{MBq}{ }^{90}$ Y-DOTA-octreotide yielded a dose ranging from $48 \mathrm{~Gy}$ (1-g tumour) to $60 \mathrm{~Gy}$ (10-g tumour) and $2 \times 277.5 \mathrm{MBq}{ }^{177} \mathrm{Lu}$-DOTA,Tyr ${ }^{3}$-octreotate yielded a dose of $58 \mathrm{~Gy}$ to a tumour from 1 to $10 \mathrm{~g}$ independent of size. The dose model used was a spherical tumour without a cross-fire dose from other organs, which is acceptable with the shorterranged beta-particles from ${ }^{177} \mathrm{Lu}$ and the larger rat dimensions, but is prone to systematic errors for the longer-ranged $\beta$-rays from ${ }^{90} \mathrm{Y}[4]$.

\section{Dose-effect relations for tumour control}

It is also possible to use ${ }^{111}$ In-labelled peptides to study doseeffects both in tumours $[9,10]$ as well as in normal tissue [11]. The conversion and Auger electrons in the spectrum of ${ }^{111} \mathrm{In}$ are capable of creating doses to the tumours in the curable range but also doses to the kidneys near the threshold for renal damage. It has been shown that the survival of rats with subcutaneously implanted CA20948 tumours with a size less than $1 \mathrm{~cm}^{2}$ could be prolonged and the prolongation time was activity-dependent. In larger tumours no prolongation of survival was seen. Radiation doses to the tumours of about 10 Gy were achieved, but unfortunately no specific dosimetry was performed, so this dose forms a crude estimate. In another study in rats, the doubling time of implanted CA20948 tumours was found to be $8 \pm 1$ days [12].

Apparently tumour doses larger than $10 \mathrm{~Gy}$ are needed for cure of rat CA20948 tumours. This dose is much lower than the therapeutic doses needed clinically as shown by Pauwels et al. [13]. Tumour shrinkage after ${ }^{90}$ Y-DOTA-octreotide therapy in patients with neuroendocrine tumours shows only a clear correlation with radiation doses above $120-150 \mathrm{~Gy}$. It is therefore of great importance to set up preclinical radiation dosimetry experiments that clarify the relationships between radiation dose, response and tumour cell properties. Radiation sensitivity, growth pattern and heterogeneity of tumour (uptake) are important cell properties to be investigated, as they influence tumour control [14]. The radiation sensitivity, tumour growth and heterogeneity of neuroendocrine tumours in humans are different from the preclinical therapy model [15], but when the conditions for each setting are known predictive preclinical therapy research can be performed with any radionuclide emitting low-LET radiation.

\section{Relation tumour size and beta-particle range}

In ground-breaking studies by O'Donoghue et al. [16] and Nahum [17], it was shown that dose, particle range and tumour size are important factors that define the probability of cure with radionuclides. Important factors in the response and growth of tumour cells are the radiosensitivity $\alpha$ and the tumour doubling time $T_{\mathrm{D}}$. The surviving numbers of clonogenic cells after irradiation follow the linear quadratic (LQ) model. The quadratic term is neglected as this term expresses the component of repairable damage to cells which is negligible with low dose-rate exposure [18], and hence a linear exponential relationship with the dose is given by:

$$
\begin{aligned}
S=\exp (-\alpha E D), \text { with }: E & =1-\frac{\gamma}{\alpha R_{0}}\left\{1-\ln \left(\frac{\gamma}{\alpha R_{0}}\right)\right\}, \\
\text { when } R(t) & >\frac{\gamma}{\alpha}, \text { else } 0 .
\end{aligned}
$$

where the dose $D$ is given by a monoexponentially decaying dose rate $R$ with (effective) decay constant $\lambda\left(=\ln (2) / T_{1 / 2}\right)$ and initial dose rate $R_{0}$, according to $R(t)=R_{0} \exp (-\lambda t)$. The effective tumour repopulation constant $\gamma\left(=\ln (2) / T_{\mathrm{D}}\right)$ in the dose wasted-by-proliferation term $E$ only works when the dose rate remains larger than $\gamma / \alpha$. After in-vitro irradiation of CA20948 cells with ${ }^{131}$ I an almost linear cell survival dose-effect curve was found, whereas following exposure to X-rays survival showed the typical LQ model curvature (Fig. 1) [19]. The parameters of the LQ model curve fitted to these data yielded: $\alpha=0.3 / \mathrm{Gy}$ (X-rays) to $0.35 / \mathrm{Gy}\left({ }^{131} \mathrm{I}\right)$. The difference between the found $\alpha$-values was not significant, but the difference in curvature was. On exposure to X-rays the curvature could be expressed by an $\alpha / \beta$ ratio of $8.3 \mathrm{~Gy}$, and exposure to ${ }^{131} \mathrm{I}$ by an $\alpha / \beta$ ratio of $102 \mathrm{~Gy}$. This straightening out of the LQ curve can be explained by almost complete repair of the sublethal damage, but could also be an indication of the $\mathrm{LQ}(\mathrm{L})$ model, as used to explain responses to hypofractionated radiotherapy [20]. Contrary to the general idea, large $\alpha / \beta$ ratios can also occur in tumours that repopulate quickly [21]. 
CA20948

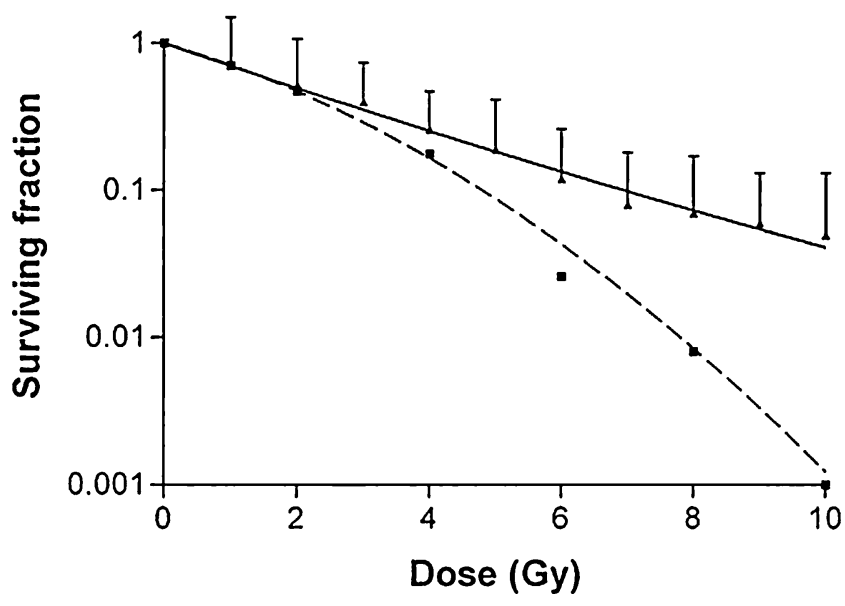

Fig. 1 Clonogenic survival curve for CA20948 using two methods: (1) external-beam therapy (dashed line), and (2) radionuclide therapy (solid line). The data presented are the means \pm standard deviation from at least three independent experiments (taken from reference [15] with permission of the publisher)

\section{Linear Quadratic model for tumour control}

Repopulation of clonogenic tumour cells escaping cell kill during and after radiotherapy is of great concern in protracted therapy schemes, such as low dose-rate brachytherapy and possibly also radionuclide therapy. The hypothesis that tumours always have high a $\alpha / \beta$ ratio has been found not to hold with all types of cancer, for that reason Eq. 1 can be adjusted to include the quadratic term $[22,23]$ :

$S=\exp \left(-\alpha D-G \beta D^{2}+\gamma T\right)$

where $G$ is the dose protraction factor accounting for sublethal damage repair during radiation exposure, $\alpha$ and $\beta$ represent the linear and quadratic intrinsic radiosensitivity, respectively, and $\gamma$ is the tumour repopulation rate as defined in Eq. 1. For the monoexponential decaying dose rate, the treatment time $T$ is defined as the time during which the dose rate remains larger than $\gamma / \alpha$ and hence the number of cells killed by radiation outweighs the number of cells from tumour growth:

$D=\frac{R_{0}}{\lambda}(1-\exp (-\lambda T))$,

$G=\frac{2 R_{0}^{2}}{D^{2}(\mu-\lambda)}\left[\frac{1}{2 \lambda}(1-\exp (-2 \lambda T))-\frac{1}{\mu+\lambda}(1-\exp (-(\mu+\lambda) T)]\right.$,

$T \approx-\frac{1}{\lambda} \ln \left(\frac{\gamma}{\alpha R_{0}}\right)$

where $\mu$ is the rate of repair of sublethal damage in the tumour cells $\left(=\ln (2) / T_{\text {rep }}\right)$ and the median tumour repair half-life $T_{\text {rep }}$ is $60 \mathrm{~min}$ [24]. The repair half-life for an intermediate risk group of prostate cancer was derived from clinical data at $T_{\text {rep }}=$ $16 \mathrm{~min}$ [23]. No study has been performed on the repair halflife of neuroendocrine tumours or of CA20948 tumours.
The probability of local control of a tumour treated by radiotherapy is thought to depend on the number of surviving tumour cells according to Poisson statistics. The tumour control probability (TCP) is therefore defined as a function of $S$ as:

$T C P=\exp (-K S)$

where $K$ stands for the initial number of clonogenic tumour cells and depends strongly on its risk level. O'Donoghue assumed for a 1 -g tumour a $K$ of $3 \times 10^{7}$ clonogenic cells [16]. This magnitude of clonogen density would correspond to a high risk (aggressive) type of tumour [24, 25]. An intermediate risk group of prostate cancers with a clonogen cell density of $3 \times$ $10^{6}$ cells/g was chosen in the TCP model by Wang and Li [23], and this choice was followed in this study, as well as higher cell densities. Tumour repopulation after irradiation is delayed by a lag or kick-off time $T_{\mathrm{k}}$, which could be as long as 40 days for CA20498 [12]. Prostate cancer (PC-3) tumourbearing mice in response to a low dose of 14 Gy of the ${ }^{177} \mathrm{Lu}-$ labelled bombesin analogue AMBA showed a progression-free survival of 37 days [26]. Both the tumour growth curves for PC-3 after treatment with ${ }^{177} \mathrm{Lu}$ AMBA and for CA20948 after treatment with ${ }^{111}$ In-DOTA-octreotate [9] show a lag time of approximately 21 days. This lag time was incorporated into the tumour cell survival by replacing $T$ by $T-T_{\mathrm{k}}$ in Eq. 2 .

\section{Application of LQ model in peptide receptor radionuclide therapy}

The consequence of the radiation sensitivity of $\alpha=0.35 / \mathrm{Gy}$ for cell survival as a function of dose without cell growth is shown in Fig. 2. When the dose is calculated with an error of $10 \%$, which would be outstanding in radionuclide dosimetry, the $95 \%$ confidence interval for cell survival after receiving a dose of 50 Gy would range between 1.24 and 0.005 cells for a 1 -g tumour with $3 \times 10^{6}$ tumour cells. This represents a difference between no response and cure, as expressed with the TCP, which ranges between $1 \%$ and $98 \%$. This huge amplification of the dose shows the necessity for obtaining high accuracy in dosimetry measurements. To obtain this, the accuracay of all dose aspects should be improved: the kinetics of the radiolabelled compound, determination of tumour volume and calculation of the radiation transport. The cell survival curve is linear exponential with one-log of cell kill after $D_{0}=2.9 \mathrm{~Gy}$. To obtain certain cures the dose should be $>61$ Gy for a 0.01-g tumour, >78 Gy for a 1-g tumour and $>94$ Gy for a 100-g tumour. When considering the dose effect of peptide receptor radionuclide therapy that was observed in rats, the uptake kinetics also need to be incorporated. The kinetic data for ${ }^{177}$ Lu-DOTA-octreotate [8] was used with an uptake in the tumour of $0.96 \pm 0.15 \% / \mathrm{g}$ and uptake half-life of $14 \pm 19 \mathrm{~min}$ and a clearance half-life of $34 \pm 6 \mathrm{~h}$. 


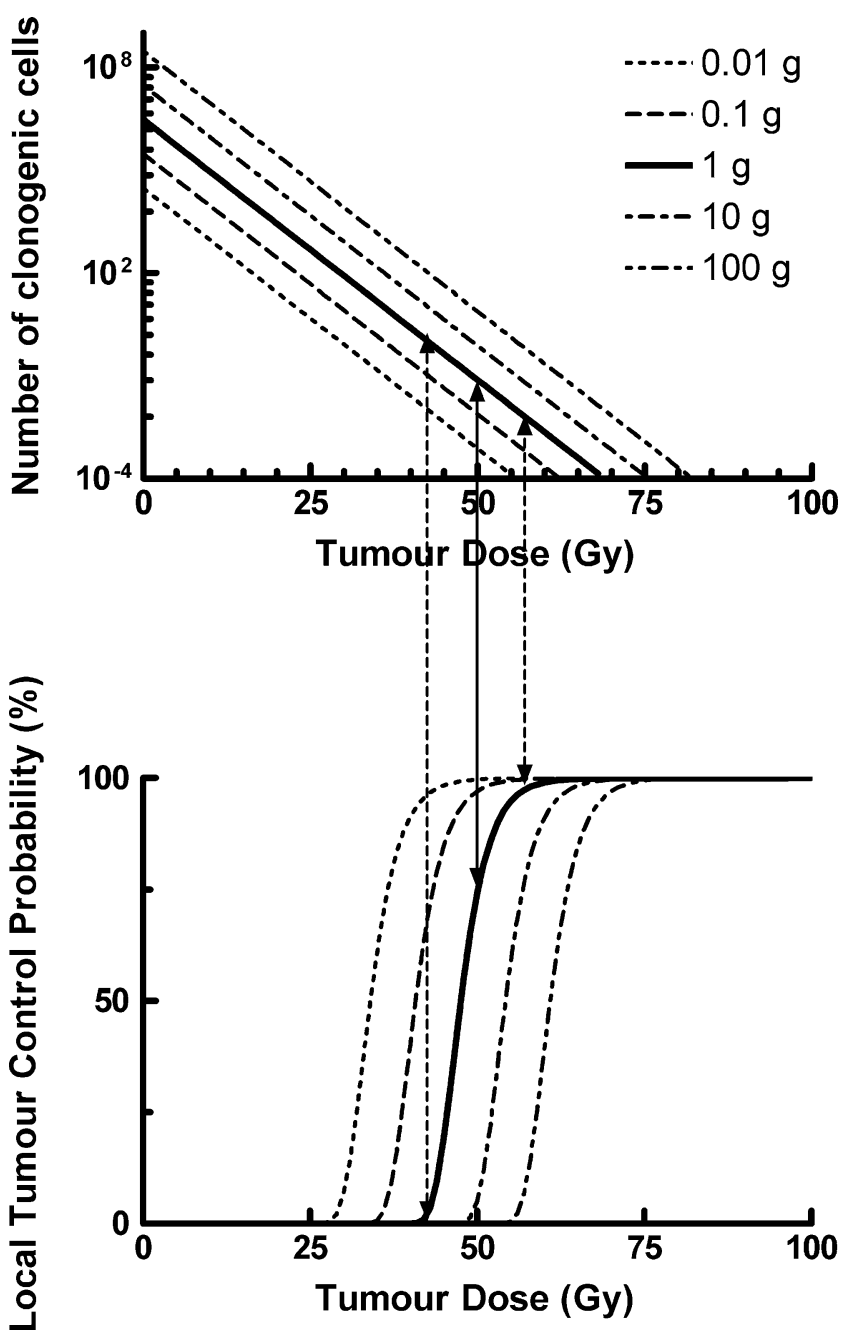

Fig. 2 Theoretical tumour cell survival curves for CA20948 tumour cells (top graph) and local tumour control probability (TCP, bottom graph) as a function of dose for five tumour weights. The arrows show the $95 \%$ confidence intervals with $10 \%$ uncertainty in a dose of 50 Gy in a 1 -g tumour producing a TCP of $74 \%$ (95\% CI $1-98 \%)$. Radiation sensitivity $\alpha=0.25 / \mathrm{Gy}$, clonogenic cell density $3 \times 10^{6}$ cells $/ \mathrm{g}$, and tumour cell doubling time $T_{\mathrm{D}}=4$ days

The difference between the three radionuclides in terms of cell survival, as shown in Fig. 3, is striking, especially since for ${ }^{177} \mathrm{Lu}$ the maximum activity of $555 \mathrm{MBq}$ was not analysed, whereas for the other two the maximum activity was used. Only ${ }^{177} \mathrm{Lu}$ is capable of creating a high enough cell kill in tumours of all sizes, although its effect is reduced in the larger tumours. The low-energy electron spectrum from ${ }^{111} \mathrm{In}$ produces curable conditions in the smallest tumours, but its effect diminishes in tumours of intermediate size. The increase in cell kill in the larger tumours is a result of its gamma rays. As expected, only larger tumours respond well to ${ }^{90} \mathrm{Y}$. The differences in dose-response for the radionuclides can be explained by the differences in dose (in a 1-g tumour: ${ }^{90} \mathrm{Y}$ $46 \mathrm{~Gy},{ }^{111} \mathrm{In} 3 \times 14 \mathrm{~Gy}$, and $\left.{ }^{177} \mathrm{Lu} 64 \mathrm{~Gy}\right)$. The fractionation of ${ }^{111}$ In helps to shift regrowth to later time-points.
The robustness of the dose-response models can be tested by varying their parameters. Reducing the tumour cell repopulation lag time to 10 days has little effect (Fig. 4; only the duration of the cure is reduced), but at lag times less than 5 days the TCP following irradiation with ${ }^{111} \mathrm{In}$ is eliminated as a result of repopulation of the tumour during the intervals between the therapy cycles with which ${ }^{111}$ In is given. As this repopulation has not been observed with a lag time longer than 10 days, it was set at 14 days. Raising the clonogenic cell density by a factor of 10 , to $3 \times 10^{7}$, will considerably reduce the TCP with ${ }^{90} \mathrm{Y}$ and ${ }^{111} \mathrm{In}$ (Fig. 5). For example, raising the clonogenic cell density by a factor of 10 in a 10-g tumour will reduce the TCP of ${ }^{90} \mathrm{Y}$ from $88 \%$ to $28 \%$, but as cures were observed in the larger tumours a clonogen density of $3 \times 10^{6}$ cells/g seems to be a reasonable estimate. Remarkably, ${ }^{177} \mathrm{Lu}$ is able to cure small and mediumsize tumours over the whole range of clonogen density. Varying the tumour repopulation time $T_{\mathrm{D}}$ has no effect on the TCP, but only on the time to regrowth. The linear radiation sensitivity factor $\alpha$ has a direct influence on the TCP. With ${ }^{177} \mathrm{Lu}$ it is possible to cure a 1-g tumour with radiation sensitivities above $0.28 / \mathrm{Gy}$, whereas with ${ }^{90} \mathrm{Y}$ this threshold is $0.38 / \mathrm{Gy}$ (Fig. 6a). In smaller tumours, the threshold for ${ }^{177} \mathrm{Lu}$ is even lower $(0.22 /$ Gy). Decreasing the $\alpha / \beta$ ratio from 103 Gy to 10 Gy has no influence on the TCP of ${ }^{177} \mathrm{Lu}$, but slightly increases the TCP of ${ }^{90} \mathrm{Y}$, and also increases that of ${ }^{111}$ In (Fig. 6b). Increasing the repair half-life from $16 \mathrm{~min}$ to $2 \mathrm{~h}$ affects in particular the TCP of ${ }^{90} \mathrm{Y}$ : in a 1 -g tumour the TCP increases from $81 \%$ to $88 \%$. Increasing the repair half-life has little effect on either ${ }^{177} \mathrm{Lu}$ or ${ }^{111} \mathrm{In}$. This is because of their lower dose rates which are a consequence of the longer half-life of ${ }^{177} \mathrm{Lu}$ and the fractionation of the dose of ${ }^{111} \mathrm{In}$. With lower $\alpha / \beta$ ratios the effect of increasing the repair half-life $T_{\mu}$ would be greater.

As injected activity is the most easily measured quantity, TCP as a function of injected activity, as shown in Fig. 7, illustrates much of the preclinical findings for radionuclide therapy efficacy. With ${ }^{90} \mathrm{Y}$ cure of tumours less than $1 \mathrm{~g}$ in weight is only possible at high activities, which are impossible to administer in one shot. At a feasible dose of $370 \mathrm{MBq},{ }^{90} \mathrm{Y}$ can cure tumours from 1 to $100 \mathrm{~g}$ in weight. Three shots of ${ }^{111}$ In can cure tumours of the lowest weight considered $(0.01 \mathrm{~g})$, as well as tumours above $1 \mathrm{~g}$. Only ${ }^{177} \mathrm{Lu}$ is able to cure tumours over the whole weight range considered.

The radiation doses to the tumour and organs are not always homogeneously distributed. With a gaussian distributed variation (with standard deviation sigma) in the dose over the tumour the homogeneous $\mathrm{TCP}_{\text {hom }}$ can

Fig. 3 Theoretical survival curves of clonogenic cells and consequent TCP in $0.01-\mathrm{g}, 0.1 \mathrm{-g}, 1 \mathrm{-g}$ and $10 \mathrm{~g}$ tumours after irradiation with $370 \mathrm{MBq}{ }^{90} \mathrm{Y}, 370 \mathrm{MBq}{ }^{111}$ In three times, and $277.5 \mathrm{MBq}{ }^{177} \mathrm{Lu}$, all according to the tumour uptake kinetics observed with ${ }^{177} \mathrm{Lu}$ DOTAoctreotate in rats bearing CA20948 tumours 

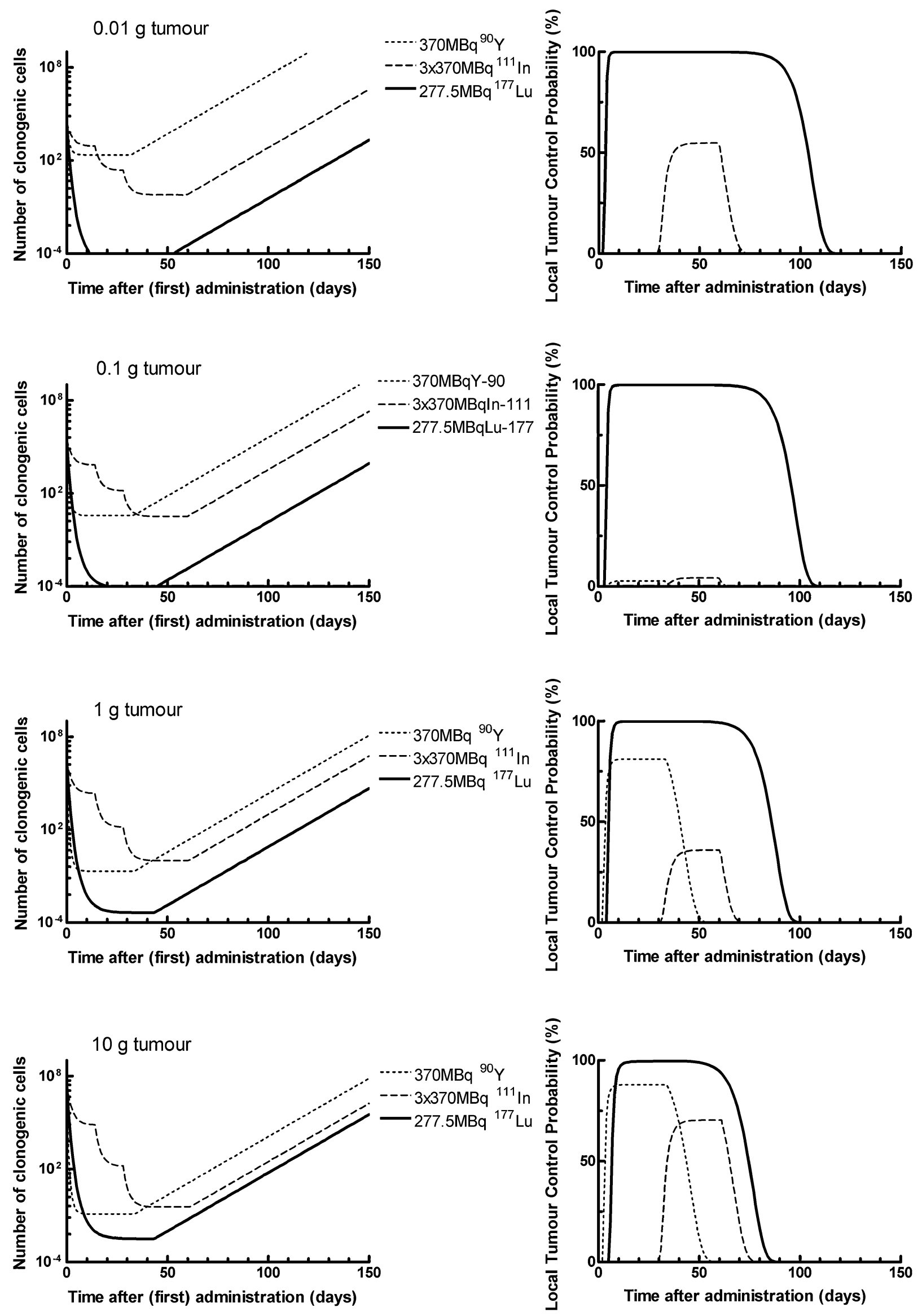


\section{1 gram tumour}

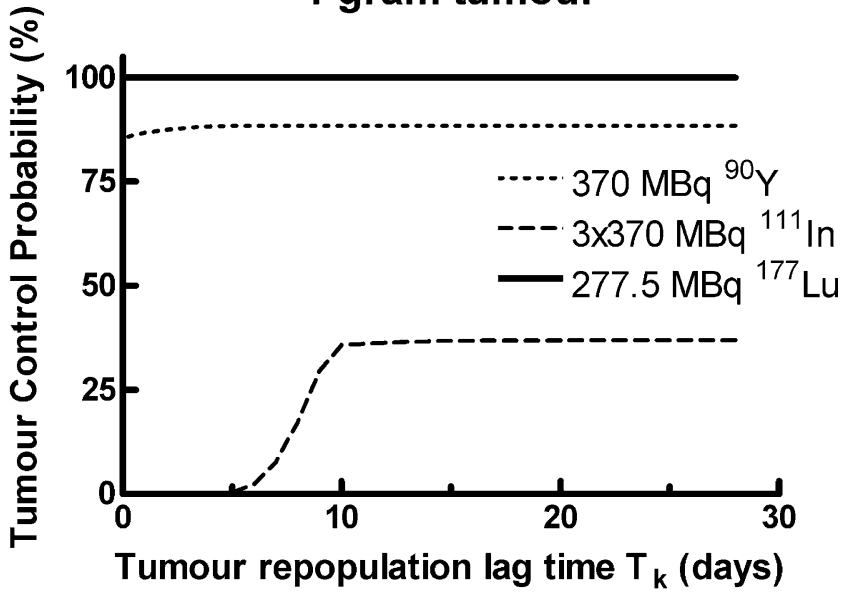

Fig. 4 Theoretical TCP for a 1-g tumour as a function of tumour cell repopulation lag time $T_{\mathrm{k}}$ after exposure to peptides labelled with ${ }^{90} \mathrm{Y}$, ${ }^{111}$ In or ${ }^{177} \mathrm{Lu}$

be adjusted according to $[27,28]$ : $T C P_{h e t}(D, \sigma)=$ $\int_{0}^{\infty} \exp \left(-\frac{(\Delta-D)^{2}}{2 \sigma^{2}}\right) T C P_{\text {hom }}(\Delta) d \Delta$. The resulting graphs are shown in Fig. 8. A variance in the radiation dose to the tumour of $50 \%$ reduces tumour control with the high-dose administrations (555 and $277.5 \mathrm{MBq}$ ) 100 days after administration from $100 \%$ to $60-90 \%$, whereas low doses only show therapeutic effects when the variance in the dose is high enough. The equivalent uniform dose is the uniformly distributed dose, or biologically effective dose, that produces an equivalent cell kill to the actual nonuniformly distributed dose [27].

\section{Discussion and conclusion}

It is possible to use dosimetry to guide preclinical therapy studies. Dose-effect curves for tumour cell kill should be obtained with the highest possible accuracy, as the steepness of the curve amplifies the uncertainty of the outcome. As ${ }^{111} \mathrm{In}$ is superior for imaging and offers the possibility of good quantitation, this radionuclide could be used successfully to study dose-effects of radionuclide therapy as a model for other low-LET radiation emitters. The Auger electron part of its spectrum may form a high-LET exposure, but only when incorporated into the cell nucleus. Most peptides remain on the cell membrane or in the cytoplasm and do not penetrate into the nucleus. The problem with using ${ }^{111}$ In for therapeutic doses is its limited specific activity disabling high activity labelling of low peptide amounts. This problem can be circumvented by administering multiple doses.

The fundamental radiation sensitivity properties of the tumour models in preclinical therapy should be determined. Studies should identify the lag time in repopulation and tumour doubling time after radionuclide exposure. The
$370 \mathrm{MBq}{ }^{90} \mathrm{Y}$-DOTA-octreotide
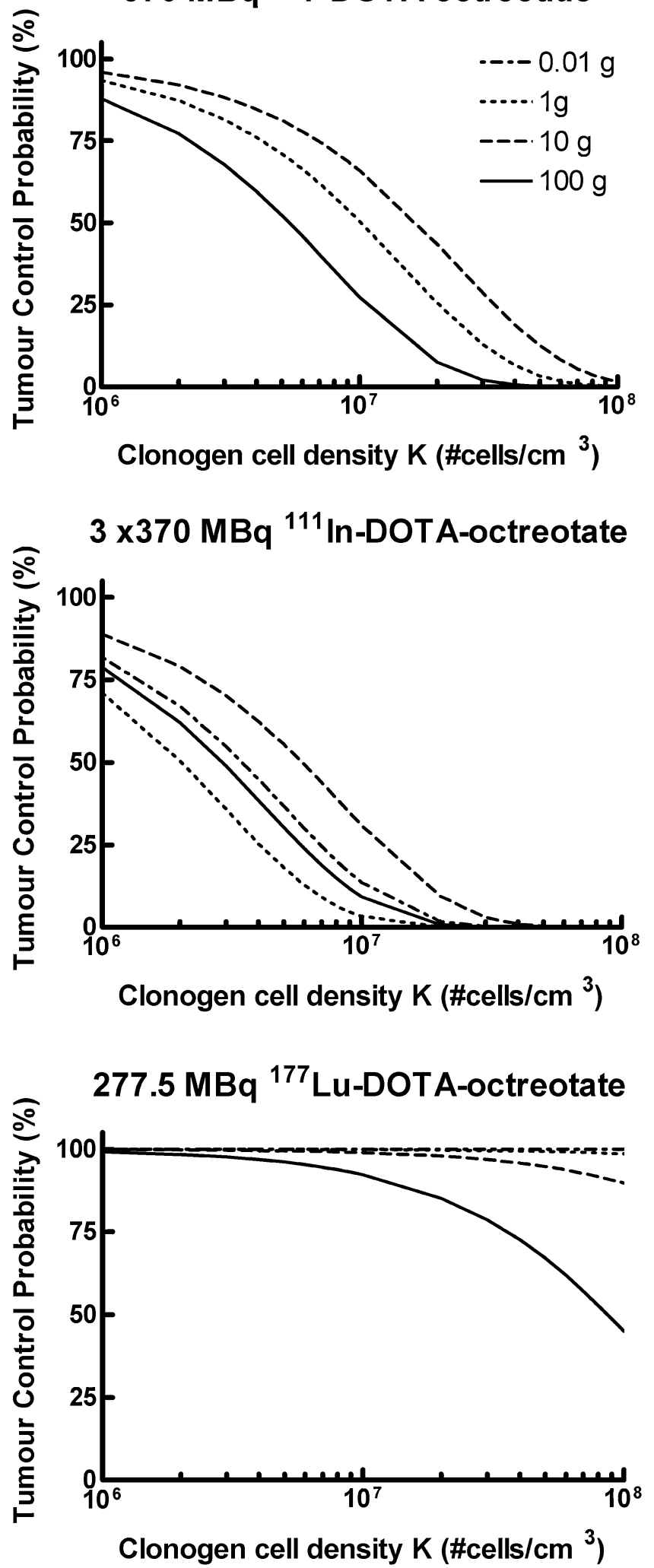

Fig. 5 Theoretical TCP of CA208948 tumours in rats as a function of clonogenic cell density $K$ for four tumour weights $(0.01,1,10$ and $100 \mathrm{~g}$ ) and three radionuclides 


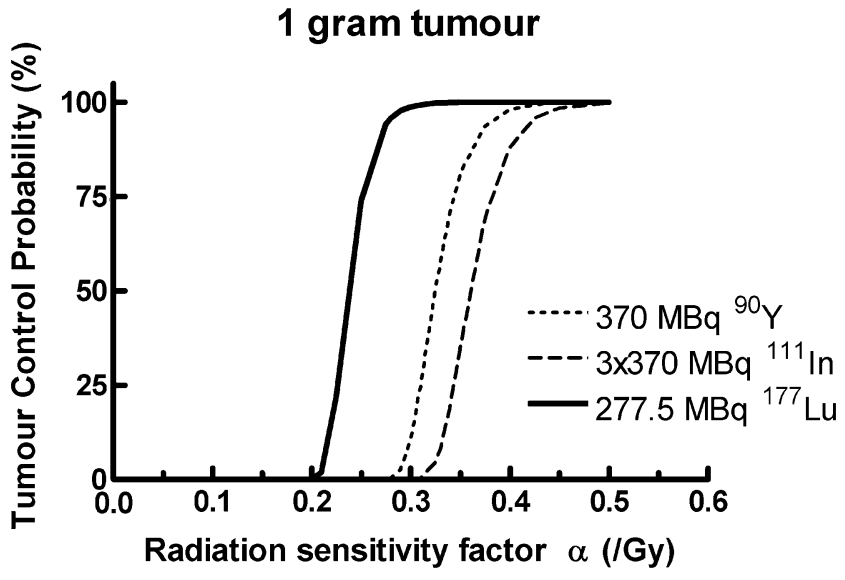

1 gram tumour
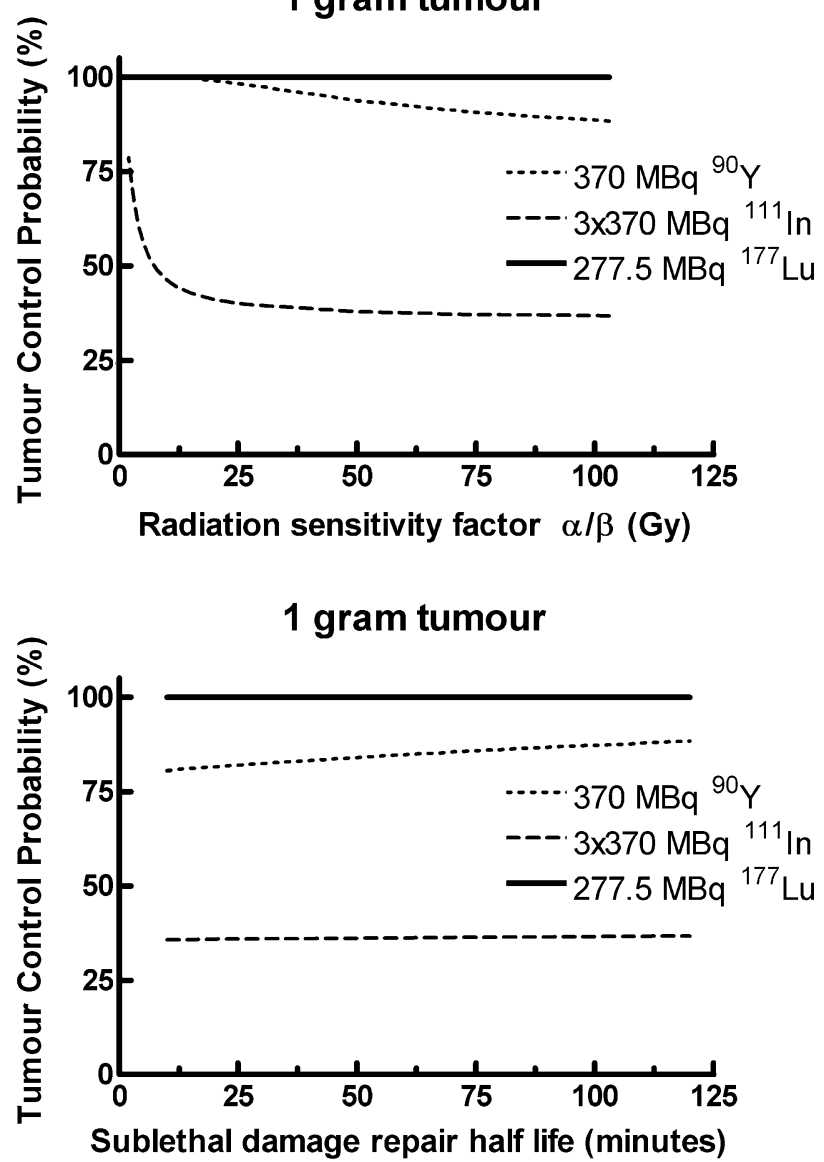

Fig. 6 Theoretical TCP of 1 -g CA208948 tumours in rats as a function of radiation sensitivity parameters according to the LQ model: linear coefficient $\alpha$, curvature $\alpha / \beta$ and repair half-life of sublethal damage $T_{\mu}$ for three radionuclides

repopulation lag time after giving a radiation dose may obscure the effect of variations in tumour repopulation, which can cause therapy resistance [29]. Radiation sensitivity parameters $\alpha$ and possibly the curvature $\alpha / \beta$ should be determined, with the appropriate repair half-life. Clonogenic cell density plays an important role in the tumour cure models, but implanted tumours are most probably
${ }^{90}$ Y-DOTA-octreotide

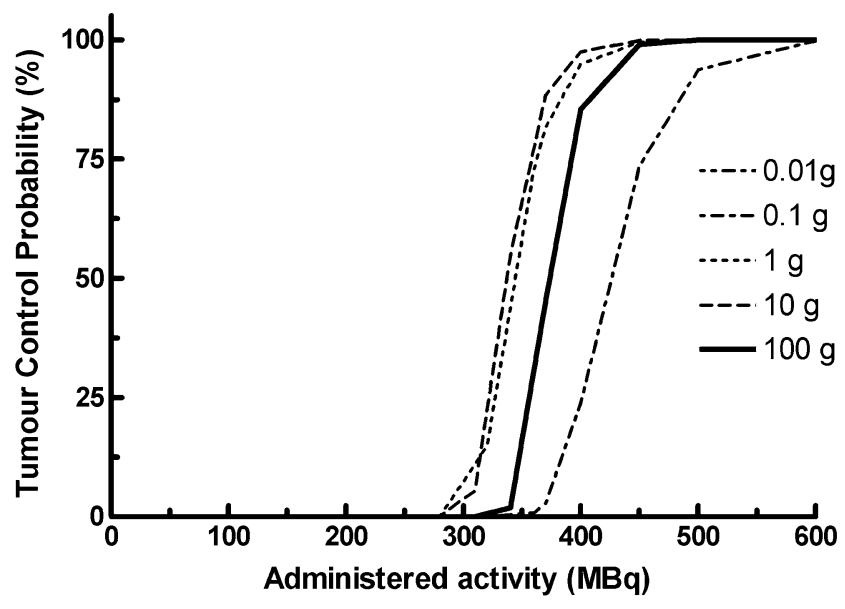

3 cycles ${ }^{111}$ In-DOTA-octreotate

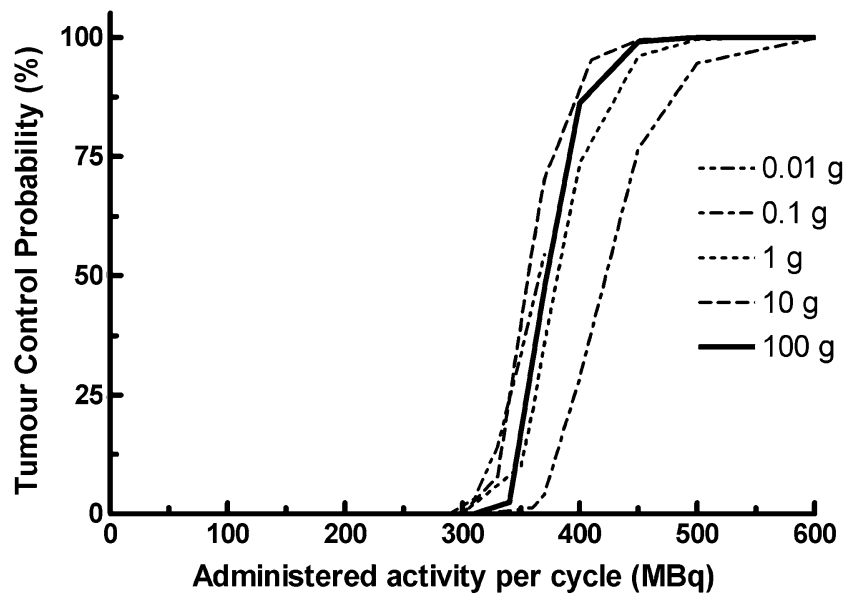

${ }^{177}$ Lu-DOT A-octreotate

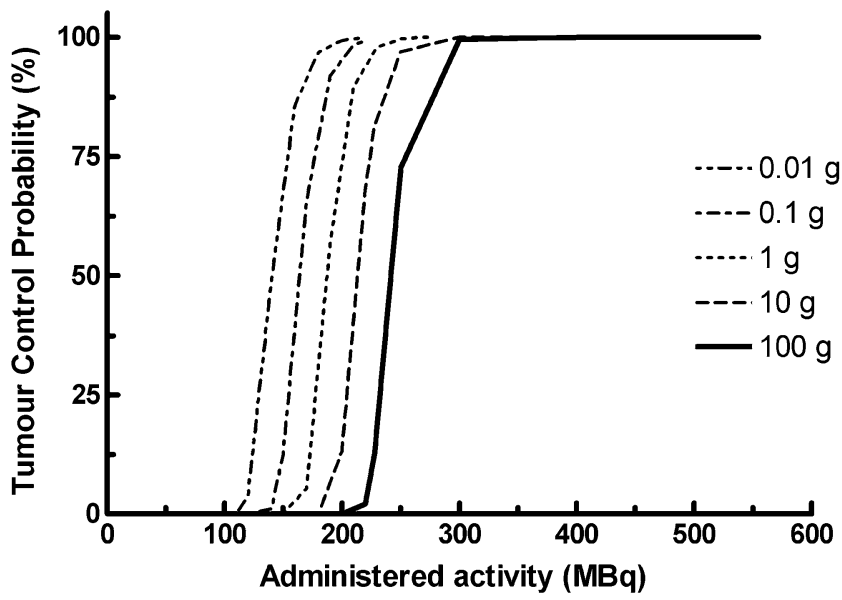

Fig. 7 Theoretical TCP as a function of administered activity, with $3 \times 10^{6}$ clonogenic cells $/ g, \alpha=0.35 / \mathrm{Gy}, \alpha / \beta=103 \mathrm{~Gy}, T_{\mu}=16 \mathrm{~min}, T_{\mathrm{k}}=14$ days, tumour uptake $0.96 \pm 0.15 \% / \mathrm{g}$ and clearance half-life of $34 \pm 6 \mathrm{~h}$ 


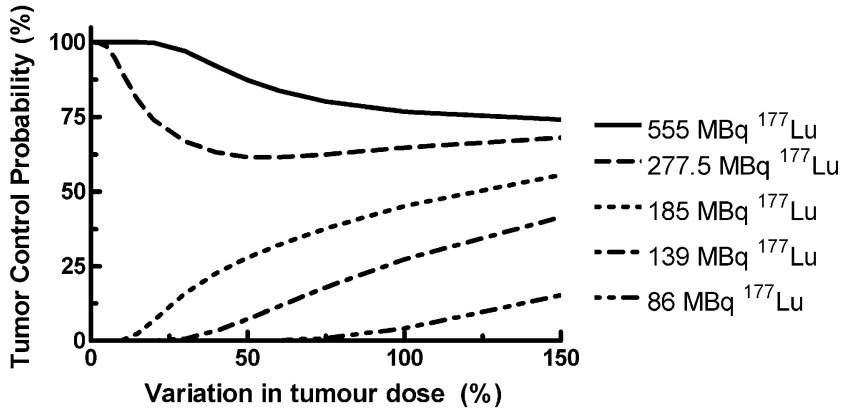

Fig. 8 Theoretical TCPs on day 100 for 1-g CA20948 implanted tumours as a function of relative variation in radiation dose to the tumour volume for ${ }^{177} \mathrm{Lu}$-DOTA-octreotate administered with the following activities and mean tumour doses: $555 \mathrm{MBq}$ (129 Gy), $277.5 \mathrm{MBq}$ (65 Gy), $185 \mathrm{MBq}$ (43 Gy), $139 \mathrm{MBq}$ (32 Gy), and $86 \mathrm{MBq}(20 \mathrm{~Gy})$

much more aggressive than neuroendocrine tumours in the clinic. This might induce a conceptual error in preclinical research into these tumours, as aggressive tumours tend to show more direct effects than those growing more slowly. This might indicate beneficial effects of fractionation in reducing toxicity without reducing therapeutic efficacy. In reality neuroendocrine tumours may behave as a lateresponding tissue, where fractionation will reduce toxicity but also therapeutic efficacy [23].

Variation in the dose over the tumour [27], and also heterogeneity of the tumour itself, might prevent the observation of clear dose-effects [14, 28]. It is, however, very difficult to image heterogeneity in the activity distribution over the tumour, unless ex-vivo autoradiography or a high-resolution (pinhole) camera can be used. Heterogeneity in tumour cell kinetics as a result of variation in clonogenic cell density [14], cell growth [29] or radiation sensitivity for example because of hypoxia is also difficult to measure, especially since all sources of variation will occur simultaneously and show interactions. Because of these variations, tumours smaller than $1 \mathrm{~cm}^{2}$ receiving peptide radiotherapy with $277.5 \mathrm{MBq}{ }^{177}$ Lu-DOTA-octreotate show regrowth in $25 \%$ of the animals, despite the TCP of $100 \%$ based on the mean radiation dose. The fact that tumours treated with an activity of $555 \mathrm{MBq}$ do not show regrowth shows that the variations in dose and other parameters are less than 50\% [8]. The large uncertainty in the dose to these tumours, however, unfortunately prevents the use of these data outside this empirical setting. However, it was shown by Buffa and Nahum that the typical (radio)biological heterogeneity in tumours has more effect on tumour control than uncertainty in the dose or dose distribution [30].

It is possible to use ${ }^{111}$ In as a surrogate for testing clinical efficacy of a radiolabelled therapeutic pharmaceutical in preclinical research, although the therapy should be designed on an absorbed dose basis instead of the generally applied activity scheme. Its reduced therapeutic efficacy in tumours of intermediate size impedes easy interpretation, which is not a factor in therapy experiments based on ${ }^{177} \mathrm{Lu}$. Dosimetrybased tumour control studies involve determination of many radiobiological parameters which are implicitly followed when using the actual therapy radionuclide itself, and enhance the science of the dose-effect by radionuclide therapies beyond the empirical knowledge.

Open Access This article is distributed under the terms of the Creative Commons Attribution Noncommercial License which permits any noncommercial use, distribution, and reproduction in any medium, provided the original author(s) and source are credited.

\section{References}

1. Keenan MA, Stabin MG, Segars WP, Fernald MJ. RADAR realistic animal model series for dose assessment. J Nucl Med. 2010;51:471-6.

2. Kolbert KS, Watson T, Matei C, Xu S, Koutcher JA, Sgouros G, et al. Factors for liver, spleen and kidney. J Nucl Med. 2003;44:784-91.

3. Bitar A, Lisbona A, Thedrez P, Sai Maurel C, Le Forestier D, Barbet $\mathrm{J}$, et al. Voxel-based mouse for internal dose calculations using Monte Carlo simulations (MCNP). Phys Med Biol. 2007;52:1013-25.

4. Hui TE, Fisher DR, Kuhn JA, Williams LE, Nourigat C, Badger CC, et al. A mouse model for calculating cross-organ beta doses from yttrium-90-labeled immunoconjugates. Cancer. 1994;73(3 Suppl):951-7.

5. de Jong M, Breeman WA, Bernard BF, van Gameren A, de Bruin E, Bakker WH, et al. Tumour uptake of the radiolabelled somatostatin analogue [DOTA0,TYR3] octreotide is dependent on the peptide amount. Eur J Nucl Med. 1999;26:693-8.

6. Behr TM, Blumenthal RD, Memtsoudis S, Sharkey RM, Gratz S, Becker W, et al. Cure of metastatic human colonic cancer in mice with radiolabeled monoclonal antibody fragments. Clin Cancer Res. 2000;6:4900-7.

7. de Jong M, Breeman WA, Bernard BF, Bakker WH, Visser TJ, Kooij PP, et al. Tumor response after [(90)Y-DOTA(0),Tyr(3)] octreotide radionuclide therapy in a transplantable rat tumor model is dependent on tumor size. J Nucl Med. 2001;42:1841-6.

8. de Jong M, Breeman WA, Bernard BF, Bakker WH, Schaar M, van Gameren A, et al. [177Lu-DOTA(0),Tyr3] octreotate for somatostatin receptor-targeted radionuclide therapy. Int J Cancer. 2001;92:628-33.

9. Capello A, Krenning E, Bernard B, Reubi JC, Breeman W, de Jong M. 111In-labelled somatostatin analogues in a rat tumour model: somatostatin receptor status and effects of peptide receptor radionuclide therapy. Eur J Nucl Med Mol Imaging. 2005;32:1288-95.

10. Müller C, Schibli R, Krenning EP, de Jong M. Pemetrexed improves tumor selectivity of $111 \mathrm{In}$-DTPA-folate in mice with folate receptorpositive ovarian cancer. J Nucl Med. 2008;49:623-9.

11. Melis M, Vegt E, Konijnenberg MW, de Visser M, Bijster M, Vermeij $\mathrm{M}$, et al. Nephrotoxicity in mice after repeated imaging using 111In-labeled peptides. J Nucl Med. 2010;51:973-7.

12. Melis M, Forrer F, Capello A, Bijster M, Bernard BF, Reubi JC, et al. Up-regulation of somatostatin receptor density on rat CA20948 tumors escaped from low dose [177Lu-DOTA0,Tyr3]octreotate therapy. Q J Nucl Med Mol Imaging. 2007;51:324-33.

13. Pauwels S, Barone R, Walrand S, Borson-Chazot F, Valkema R, Kvols LK, et al. Practical dosimetry of peptide receptor radionuclide therapy with (90)Y-labeled somatostatin analogs. J Nucl Med. 2005;46 Suppl 1:92S-8. 
14. Suwinski R, Taylor JM, Withers HR. The effect of heterogeneity in tumor cell kinetics on radiation dose-response. An exploratory investigation of a plateau effect. Radiother Oncol. 1999;50:5766

15. Marcos HB, Libutti SK, Alexander HR, Lubensky IA, Bartlett DL, Walther MM, et al. Neuroendocrine tumors of the pancreas in von Hippel-Lindau disease: spectrum of appearances at CT and MR imaging with histopathologic comparison. Radiology. 2002;225:751-8.

16. O'Donoghue JA, Bardiès M, Wheldon TE. Relationships between tumor size and curability for uniformly targeted therapy with betaemitting radionuclides. J Nucl Med. 1995;36:1902-9.

17. Nahum AE. Microdosimetry and radiocurability: modelling targeted therapy with beta-emitters. Phys Med Biol. 1996;41:1957-72.

18. Stephens TC, Peacock JH, Shipley WU, Steel GG. Response to continuous irradiation (CI) in relation to the initial slope of the cell survival curve for tumours and bone marrow. $\mathrm{Br} \mathrm{J}$ Cancer. 1984;49(Suppl VI):271-4.

19. Verwijnen S, Capello A, Bernard B, van den Aardweg G, Konijnenberg M, Breeman W, et al. Low-dose-rate irradiation by 131I versus high-dose-rate external-beam irradiation in the rat pancreatic tumor cell line CA20948. Cancer Biother Radiopharm. 2004;19:285-92.

20. Borst GR, Ishikawa M, Nijkamp J, Hauptmann M, Shirato H, Bengua $G$, et al. Radiation pneumonitis after hypofractionated radiotherapy: evaluation of the $\mathrm{LQ}(\mathrm{L})$ model and different dose parameters. Int J Radiat Oncol Biol Phys. 2010;77:1596-603.

21. Fowler JF. Linear quadratics is alive and well. Int J Radiat Oncol Biol Phys. 2008;72:957-9.
22. Wang JZ, Guerrero M, Li XA. How low is the alpha/beta ratio for prostate cancer? Int J Radiat Oncol Biol Phys. 2003;55:194-203.

23. Wang JZ, Li XA. Impact of tumor repopulation on radiotherapy planning. Int J Radiat Oncol Biol Phys. 2005;61:220-7.

24. Levegrün S, Jackson A, Zelefsky MJ, Skwarchuk MW, Venkatraman ES, Schlegel W, et al. Fitting tumor control probability models to biopsy outcome after three-dimensional conformal radiation therapy of prostate cancer: pitfalls in deducing radiobiologic parameters for tumors from clinical data. Int J Radiat Oncol Biol Phys. 2001;51:1064-80.

25. Ling CC, Gerweck LE, Zaider M, Yorke E. Dose-rate effects in external beam radiotherapy redux. Radiother Oncol. 2010;95:261-8.

26. Lantry LE, Cappelletti E, Maddalena ME, Fox JS, Feng W, Chen $\mathrm{J}$, et al. 177Lu-AMBA: synthesis and characterization of a selective 177Lu-labeled GRP-R agonist for systemic radiotherapy of prostate cancer. J Nucl Med. 2006;47:1144-52.

27. O'Donoghue JA. Implications of nonuniform tumor doses for radioimmunotherapy. J Nucl Med. 1999;40:1337-41.

28. Webb S, Nahum AE. A model for calculating tumour control probability in radiotherapy including the effects of inhomogeneous distributions of dose and clonogenic density. Phys Med Biol. 1993;38:653-66.

29. Malinen E, Freykowski S, Kummermehr J. Regrowth delay analysis of irradiated tumors in the curative dose region. Int $\mathrm{J}$ Radiat Oncol Biol Phys. 2000;46:173-7.

30. Buffa FM, Nahum AE. Monte Carlo dose calculations and radiobiological modeling: analysis of the effect of the statistical noise of the dose distribution on the probability of tumour control. Phys Med Biol. 2000;45:3009-23. 